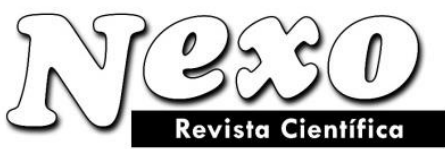

Vol. 34, No. 01, pp. 280-309/Marzo 2021
ISSN-E 1995-9516

Universidad Nacional de Ingeniería COPYRIGHT () (UNI). TODOS LOS DERECHOS RESERVADOS http://revistas.uni.edu.ni/index.php/Nexo https://doi.org/10.5377/nexo.v34i01.11306

\title{
Proof of Euclid's fifth postulate and the establishment of the metaphysical foundations of the so-called space (metametry)
}

\section{Demonstración del quinto postulado de Euclides y creación de las bases metafísicas del llamado espacio (metametría)}

\author{
Eduard O. Heyfetz \\ Odessa National I.I. Mechnikov University, Biology Department, Ukraine. \\ * Heyfetz.Eduard@gmail.com \\ (recibido/received: 19-November-2020; aceptado/accepted: 18-January-2021)
}

\begin{abstract}
Euclid's fifth postulate has been accepted as a theorem since the time of ancient Greece. The efforts to prove it have been going on for nearly 2000 years. Non-Euclidean geometry, based on its rejection, emerged in the first half of the 19th century. The author of the present article returns to the problem by addressing the metaphysical foundations of physics. The author has found the ideal instrument for analyzing infinity to be an infinitely small unit, which cannot be divided further. With the help of this instrument, the fundamental properties of the so-called space were found. It was concluded that there are no oblique or curved lines on the basic level. The apparent curved and oblique lines are stairs with negligibly fluent changing or constant steps, correspondingly. Hence, the refutation of non-Euclidean geometries and seeking a new proof of the postulate. Inter alia, it was found that the requirement to conclude the proof from Euclid's other four axioms only diverted the attention of mathematicians from the true problem. The author proved the fifth postulate on a plane. Its application to a pair of skew lines is considered. In conclusion, the author describes the basic properties of the so-called space.
\end{abstract}

Keywords: Anti-angle; Infinitely small unit; Negative angle; Parallel lines; Points of crossing; Points of opposition; Quasi-turn

\section{RESUMEN}

El quinto postulado de Euclides ha sido aceptado como un teorema desde la época de Grecia Antiga. Los esfuerzos para probarlo se han emprendido por más de dos mil años. La geometría no Euclidiana basada en el rehuso del postulado apareció en la primera mitad del siglo diecinueve. Aquí se trata de retornar al problema por medio de considerar las bases metafísicas de física. El instrumento ideal derivado aquí para analizar infinidad es una unidad infinitamente pequeña que no puede ser dividido más. Este instrumento fue usado para determinar las propiedades fundamentales del llamado espacio. Se concluyó que el nivel básico no contenía ningunas barras oblicuas o curvadas. Las barras oblicuas y curvadas aparentes son escaleras con escalones desdeñablemente fluentes variables o constantes. Por lo tanto, hay la refutación de 
las geometrías no Euclidianas y la busca por una prueba nueva del postulado. Se concluyó, entre otras cosas, que la exigencia de derivar la prueba de los otros cuatro axiomas Euclidianos desviaba la atención de los matemáticos del problema real. El autor probó el quinto postulado en una superficie plana. Se considera la aplicación del postulado a un par de líneas oblicuas. Se describen las propiedades básicas del llamado espacio para concluir el estudio.

Palabras claves: Antiángulo; Unidad Infinitamente Pequeña; Ángulo Negativo; Líneas Paralelas; Puntos De Cruce; Puntos De Oposición; Cuasiturno

\section{INTRODUCTION}

The essentials of modern geometry were set in ancient Egypt as a totality of mechanical habits. Their development is infixed in the tombs of the pharaohs - from low sloped truncate pyramids (mastabs) to stair-stepping pyramids of Joser and Hunu - the stairs of the latter were immured by order of Sneferu; from the broken pyramid of Sneferu (the angle at the base - 54o, in the upper quarter - 43o) to the wellproportioned pyramid of Khufu.

The rapid adoption of the Egyptians' achievements in ancient Greece, which experienced intensive development, gave birth to theoretical groundings. The corpus of geometry, formed by Euclid, is the fusion of geometric constructions with axiomatic proofs, which permit the binding of complicated conclusions with the basic statements via vivid demonstrations.

Correspondingly, two millennia later, acquiring spontaneous accumulations of the antique art and refuting medieval canons, the artists of the Renaissance turned to the analytical proof of human proportions plastic anatomy.

The philosophy of the ancient Greeks mainly developed from geometry. Inscribed on the portal of Plato's Academy was the sentence: "Let no one ignorant of geometry enter here." The geometrical method was considered the surest means of proving statements in both algebra and physics.

Thus, Galileo Galilei proved that objects, one of which moves with uniform speed, and the other one with uniform acceleration, will pass the same distances if the speed of the former equals half of the final speed of the latter.

\section{GEOMETRICAL DEMONSTRATIONS - EUCLID'S FIFTH POSTULATE- NON- EUCLIDEAN GEOMETRIES}

Let us construct the triangle $\mathrm{ABC}$ and let us cut it into equal segments, which correspond to equal time intervals. Line $\mathrm{AC}$ will represent time, and $\mathrm{AB}$ - speed. Let us put line $\mathrm{DF}$, parallel to $\mathrm{DC}$ through the middle of $\mathrm{AB}$ (dot $\mathrm{E}$ ), and line $\mathrm{AD}$. Rectangle $\mathrm{ADFC}$ corresponds to uniform straight motion with the speed, equal to half of the maximal speed by uniformly accelerated motion. It is obvious that SADFC = $\mathrm{SABC}$, since the segment $\mathrm{AEFC}$ is common to both figures, and the square of the triangle $\mathrm{EBF}$, belonging to $\mathrm{ABC}$ and not belonging to ADFC equals that of triangle $\mathrm{ADE}$, that belongs to ADFC and that does not belong to $\mathrm{ABC}$ (Galilei, Galileo, Works. Vol. I, p. 312) (fig. 1). 


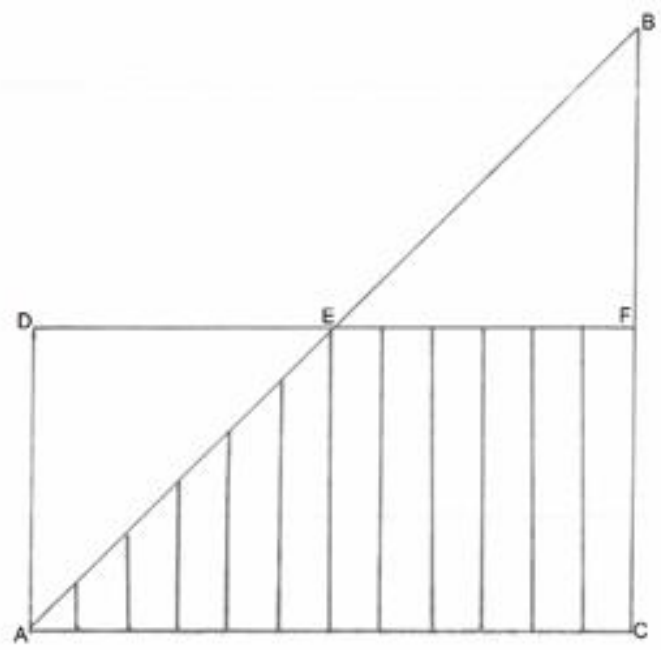

Figure 1. Geometrical Proof of Galileo Galilei

The sticking point for such an approach became Euclid's fifth postulate that was introduced as an axiom - from " $\alpha \xi_{10}$ " - veracious, in what confounded successors of the great geometer. Attempts to prove the postulate continued unsuccessfully for nearly two thousand years.

According to the given statement, "if a straight line falling on two straight lines makes the interior angles on the same side less than two right angles, the two straight lines, if produced indefinitely, meet on that side on which the angles are less than the two right angles" (Euclyd, Essentials, p. 15).

In the beginning ancient sceptics formulated a sophism, aimed to show that the point of crossing is nonreachable. Let us take two lines that approach one another. Then let us draw a segment, connecting them. Then let us divide the segment in half. Let us add a length, equal to half of the segment, to said lines. Let us mark at their end an additional segment and let us divide it anew, etc. The distance will decrease at each stage, and the lines will not arrive at the point of their crossing.

In effect, we can consider each interval between the dots as a segment. It is natural that initially we draw small distances, and then, greater ones, consisting of the former.

Furthermore, sophists proposed to diminish uninterrupted augmentations of straight lines in order to avoid their meeting, hence they recommended a clearly artificial approach. One mightemploy a simpler method: to not continue the lines or to continue them in the opposite direction.

As Proclus reasonably remarks: "That in such a simple way it is impossible to define the point of meeting, is true. But that the lines will never meet at all, is not true" (Kagan, 1975, p. $111-112$ ).

Nevertheless, the efforts of sophists led to doubt in the reliability of the fifth postulate. Apparently expressing the opinions of many geometers (among whom the famous ancient astronomer Ptolemy is mentioned), Proclus claimed: "This [fifth postulate] ought even to be struck out of the Postulates altogether; for it is a theorem involving many difficulties. Ptolemy, in a certain book, set himself the task of solving said difficulties, and it requires the demonstration of several definitions as well as theorems. And the converse of it was actually proved by Euclid himself as a theorem." (Diadokhos, Proclus, Commentary on the First Book of Euclid's "Essentials", p. 177). 
Furthermore, Proclus asserted: "The fact that, when the right angles are lessened, the straight lines converge is true and necessary; but the statement that, since they converge more and more as they are produced, they will sometime meet is plausible but not necessary, in the absence of some argument showing that this is true in the case of straight lines." (ibid., p. 178). It is remarkable that both Proclus and his followers did not try to prove this statement. In this way, cowardice bred the problem of the fifth postulate.

As a result, many attempts were made to prove the fifth postulate. In the majority of cases, there were attempts to construct it on the basis of the previous four axioms. As we can see, this approach only diverted attention from the true problem, as noted by Proclus. But then geometers felt that they proved the postulate in the correct way. It is also remarkable that according to this belief, Euclid's axioms extinguished all geometrical reality, implying the divinity of their author. Hence, we are faced with a somewhat para-religious outlook, formed through apparent difficulties.

One of the reasons, shared by many difficulties in science, is that the scientist is more inclined to overestimate, rather than underestimate, the complexity of a problem. In the case of an unsolved question, the successor often supposes that the means of their predecessors were insufficient, which may lead to the progressive and superfluous complication of the methods.

Several additional principal attempts to prove the postulate are worth mentioning. For example, Proclus proceeded from the premise that the distance between the straight lines, departing from one another, constantly grows up to infinity and that the straight line, crossing one of the parallel lines, will cross another one (ibid., p. 313); Wallis (1663) based the proof on the uniformity of triangles of an arbitrary size (Kagan, 1955, p. $46-51$ ). Bertrand (1812) suggested that if the sum of two internal angles equals two straight angles, then the part of the plane that they limit, is so small relative to the entire plane that it is contained in the latter an infinite number of times (Kagan, 1955, p. $51-54$ ). One of the latest authors of proof, the father of Yanos Bolyai, Farkas Bolyai, shows that it is possible to circumscribe a circle around each triangle (Chistiakov, 1973, p. 33. Thus, not the truth of the statement is proved, but its submission to axioms, forming the basis of geometrical thought.

Furthermore, the very methodic converts itself into an organic habit of thinking that does not provoke doubts. It is natural to seek a solution of a geometrical problem in geometry; even more so, in the geometric method that appears to be quite authentic, as arithmetic operations.

It is necessary to notice, that there were attempts to prove the postulate in more consistent ways.

While proving the main part of the fifth postulate, the author of the present article revealed a certain similarity to the approach of Nasir al-Din Tusi $(1201$ - 1274). Before the proof, he prefaced three auxiliary theorems (lemmas). It seems that the first of them is complicated, it will be mentioned later; the second and the third ones, regarding the parallelism of perpendiculars and that the sum of angles in a triangle equals $180^{\circ}$ are, presumably, excessive.

The said work was preserved as a manuscript for more than 300 years. It was published (printed) for the first time in 1594in Rome.

The shortcomings of that proof were criticized by Saccheri (1667 - 1733), who did not illuminate the proof, rather denied it (Kagan, 1955, p. 43-46). Saccheri tried to prove the postulate "by way of the contrary" but did not succeed in doing so (ibid, p. 158 - 164).

Towards the beginning of XIX century algebra, analytic geometry and physical experimentation displaced perspective geometry from natural science. Liberated from the solution of applied tasks in other sciences, 
geometry proceeds to the philosophical comprehension of its own foundations. Lobachevsky wrote: "At least, let us say that since the time of Newton and Descartes all the Mathematics, converting itself in Analytics, proceeded forward by such rapid steps, that it left far behind the teaching without which it could not stand, and which at the same time ceased to draw attention to itself, while hitherto it was of merit. In this manner, the Euclidean essentials, in spite of their deep antiquity, in spite of all the brilliant successes in Mathematics, preserved up to the present their primogenial shortcomings." (Lobachevsky, 1949, p. 185).

As a result, Euclid's fifth postulate is doubted. Furthermore, several mathematicians, Lobachevsky, Bolyai, followed closely by Gauß, broke that stream of proofs. They experimented to prove the postulate by contradiction. During that process they were so fascinated by the picture they obtained, that they saw in it not proof of the fifth postulate's importance for geometry, but the possibility to extend beyond the limits of the Euclidean geometry. In turn, elaborating on the metametric groundings of physics, and coming to conclusions incompatible with those of non-Euclidean geometry, the author was forced to return to the root of the problem, i.e., to the fifth postulate, and to comprehend the misfortunes of his predecessors, in attempting to prove the postulate.

The author of the present article believes that non-Euclidean geometry, if we compare to the conclusions of Euclid, not relatively, but in the absolute aspect, is a step backwards. If the geometry of Euclid led to the developed speculation, the authors of non-Euclidean geometry appeal to empiric observations ${ }^{1}$. If the internal infinity exists (see $\S 2$ ), such a way seems to be dead-ended.

Furthermore, with the negation of Euclid's fifth postulate, the absolute component of geometry is lost and the element of arbitrariness is introduced. Curves are considered to be straight lines in a curved space. Indeed, a straight line may be converted into a curve with the bending of the sheet on which it is drafted. Nevertheless, that line will cease to be straight one.

In a discussion with a mathematician I argued that the straight lines represent an intuitive development of mankind, and that it is necessary to find a stricter criterion for their definition. He responded that the straight lines are the pieces of a game, like chess horses, which may be formed with legs but could also be made with only a head. I think that such an approach goes beyond the frame of mathematics.

It is necessary to say, however, that Lobachevsky proposed a rational criterion of the straight line: "The straight line covers itself in all positions. I mean that during the rotation of a surface, the straight line does not alter its place, if it passes through two immobile dots on the surface." (Ibid, p. 80). This definition is too empirical. One can distinguish a straight line without rotating it. The rotation of the line does not require mention of the surface (it may be considered as a rod, hanging in the space). Accordingly, Lobachevsky's definition is not in use. Nevertheless, it reflects a real property of a straight line. It is obvious that it cannot exist in pseudosphere of Beltrami, where the geometry of Lobachevsky should work (Beltrami, 1868).

The methodological drawback of the founders of non-Euclidean geometry is striking: if they considered the fifth postulate to be unprovable, and therefore inconsistent, then contrarily they viewed their own developments as irrefutable, and therefore, as consistent. Meanwhile, the opposite is also true: the fifth postulate was not refuted, which would be the surest proof of non-Euclidean geometries. If their originators would reason in a strictly logical manner, they should conclude that the fifth postulate and their own developments are both unprovable and irrefutable. While we might take into account the inspiration of the founders of non-Euclidean geometry, such a drawback is less excusable for their followers. Their preference lies only in the naked idea of progress, which distinguishes science from religion, with its reliance on traditions. 
As Pushkin wrote, "Our well-born nobility is new // And the newer, the nobler."

Later, the idea of curved space defined the destiny of non-Euclidean geometries. Thus, Einstein, grounding the theory of general relativity, goes from physics to mathematics, considering the bending of a light beam in interstellar space as evidence of non-Euclidean metrics of the physical fields (Dafermos, Holzegel, \& Rodnianski, 2019).

Different criteria - the negation of the fifth postulate by Lobachevsky and Bolyai, operations with sets by Riemann, beam bending in Einstein's concept - imply that the question is far from its solution.

Increasing specialization does not favor it, whereas the interdisciplinary physical and mathematical character of non-Euclidean geometries is clear.

Coming back to the fifth postulate, the author of the present article considers that it is deeper than those axioms, from which his predecessors attempted to conclude it. As a result, generations of geometers raised a wall before the postulate, scrupulously patching chinks which they found. The evidence of the durability of that construction are the words of despair of F. Bolyai, addressed to his son, in which he begged him not to proceed in proving the postulate, and expressed certainty that the obscurity never will be dispersed.

Furthermore, the geometrical method permits proving the application of the statement, but not its essence. This method facilitates the study of relatively complicated figures. The world of geometrical protozoa found itself beyond the frameworks of research. Its revenge resulted in misadventures in proving the fifth postulate.

\section{THE PROOF OF EUCLID'S FIFTH POSTULATE}

Let us begin with the straight line. For the given purpose it is sufficient to use the criterion of Lobachevsky: namely that this line does not alter its shape after rotation with two immobile dots.

Angles define the degrees of convergence and divergence of straight lines. Thus, converging lines up to the point of crossing inclusively form two angles: concave (inner) and convex (outer), which are the lesser and greater sectors of a circle (fig. 2).

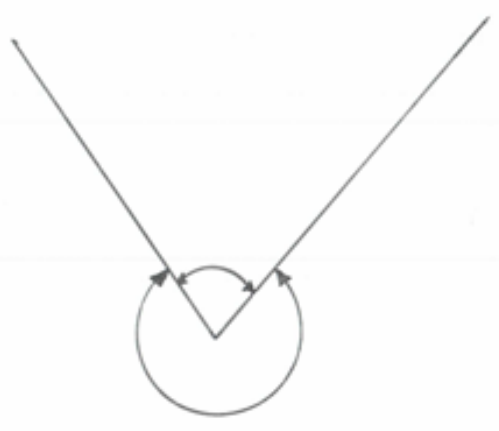

Figure 2. Inner and outer angles

As was stated above, Proclus cast doubt on the crossing of approaching lines, located on one plane. Let us prove this statement, since its obviousness was questioned by many followers of this philosopher.

In order to prove the crossing of the lines on the same plane, we can use the method, unknown to ancient geometers, in contrast to those who lived within Descartes and after him. I refer to his analytical geometry. 
Let us accept one of the lines as axis $\mathrm{x}$ or $\mathrm{y}$, and let us add the second axis, marking them. The formula which describes the straight line is $\mathrm{y}=\mathrm{ax}+\mathrm{b}$. Obviously this line crosses axis $\mathrm{y}$ at the dot that equals $\mathrm{b}(\mathrm{x}$ $=0$ ) and the other axis at the $\operatorname{dot} \mathrm{x}=0$ that corresponds to $\mathrm{x}=\frac{-b}{a}$.

The straight line $\mathrm{y}=\mathrm{c}$ crosses axis $\mathrm{y}$ at the dot $\mathrm{c}$, and passes in the distance $\mathrm{c}$ from axis $\mathrm{x}$, without approaching it. Correspondingly, $\mathrm{x}=\mathrm{d}$ will cross $\mathrm{x}$ at the dot $\mathrm{d}$, and will pass in the distance $\mathrm{d}$ from axis $\mathrm{y}$ with no approach to it.

Thus, if the straight lines are approaching on a plane, then by their continuation they should cross one another. This differs them from hyperboles, expressed by the formula $\mathrm{y}=\frac{b}{x}$, which, approaching coordinate axes, never cross them.

The author of the present paper believes that this method may prove the statement; however, it cannot reveal some of the hidden metaphysical properties of lines. In order to study them, let us return to classical geometry. A pair of straight lines is characterized by the preservation of an angle. As a result, when travelling along lines, positioned on one plane in any constant segments, the change of distance between them (or the length of segments, connecting them by a given angle ${ }^{2}$ ), is also constant.

So, as far as concerns straight lines lying on a single plane, it is true that the segment, passing between them at an equal distance, is altered by the same value. Indeed, in the opposite case, the line will deviate from the initial direction and will become a broken or curved line (fig. 3)

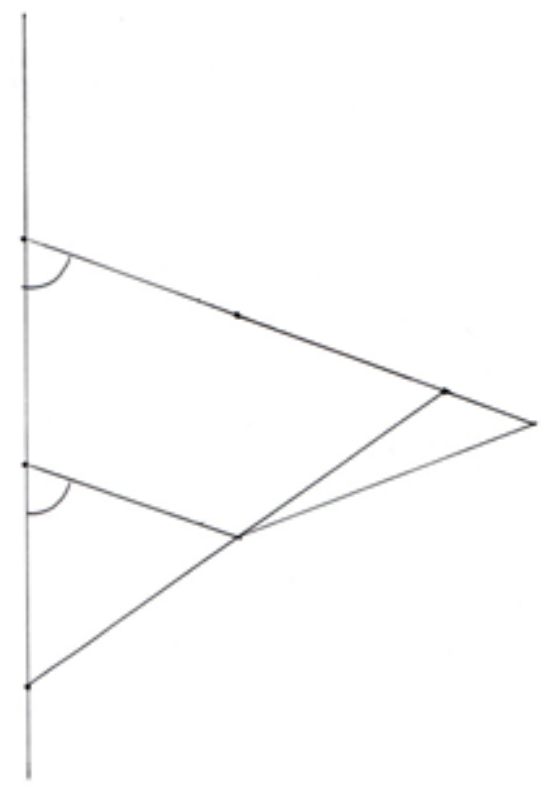

Figure 3. Constant growth of distance between straight lines

Hence, if in one direction the full length is added to the given segment, in the opposite direction it is subtracted, and the segment turns to zero, corresponding to the point of the lines' crossing.

In this way, two straight lines, located on the same plane, define the proportions of the segments, connecting them. The bottom line may be that each straight line - being a totality of dots, connected by the shortest distance - defines a contemplated dimension; whereas two lines nullify two-dimensional totality, i.e. the plane. Furthermore, this sequence is true for a bent plane, connecting the parallel ribs of a prism. 
Nevertheless, one wouldn't obtain a similar result with curves, whose significance is overestimated because they may be more complicated and more interesting for mathematicians.

In contrast, segments, connecting pair of skew lines, which may only be connected by a twisted plane, do not preserve constant proportions.

Of course, we can calculate the length of any such segment, taking into account that it represents the hypotenuse of a triangle, where one constant cathetus is the distance between one of the lines and its orthogonal projection on the plane, containing the other skew line; and the other cathetus is the line, connecting the projection with the line, lying on the same plane (the formula then is $\sqrt{ }\left(\left(1 \_0 \pm 1 \_0\right.\right.$ $[\mathrm{n}) \rrbracket^{\wedge} 2 \pm h^{\wedge} 2$ ), where lo is an initial distance between one of the lines and the orthogonal projection of the other line on the plane, containing the first line; $n$ is the amount of steps of the constant value; $h$ is the height of perpendicular, connecting one of the skew lines with its projection on the plane, where the other line lies, and the signs "plus" or "minus" alter simultaneously for both parts of the formula), but let us not forget that the projection of a skew line is an ideal notion, helping us to cognize the aforesaid dependence.

Abstracting from such auxiliary parameters, we would conclude that one of the skew lines gradually approaches the other up to a certain no-zero distance, then it turns out. Hence, we are dealing with a sort of straight line that behaves like a curve. Not in vain did French geometers call them "droites gauches" that implies both "left rights" and "clumsy straight lines".

Let us notice also that such a peculiarity is based on the proportions that still exist as distances between the projection and the line, found in the same plane, and on the constant height. Here dialectic negation, preserved negating property is present.

Another example of such negation that I would to mention is screw spiral of snails, whose asymmetry is based on two kinds of symmetry: parallel translation and turn symmetry. For this reason we appreciate their shapes.

Of course, one can imagine two skew planes, existing in the parallel three-dimensional totalities ("spaces") and between them - a "space", twisted in the fourth dimension and connecting the planes, which will behave like ones one towards the other, despite of their nature.

Nevertheless, such a twist may proceed in $n+1$-dimensional space.

As for the accepted ideas of curved space, then any true curve cannot exist without virtual straight lines, connecting its dots. The idea of the existence of curves without straight lines seems too empiric. Thus, a geographer measures the distance between remote areas, i.e., Quito and Moscow, by the curved surface of the planet, whereas a geometer, if necessary, can calculate the length of a straight chord, connecting the ends of the curve despite its empiric unavailability.

The less the outer and the greater the inner angle between defined lines, the stronger they diverge, therefore, the faster grow the lengths of a segment, placed at specific angles to the bisector. On the other hand, the greater the inner angle by an apex, the less the distance from a segment of a certain length towards the apex, the less the deviation of the lines from the segment, and the less is the sum of inner angles between the lines and the segment (fig. 4).

Thus, the greater the sum of the inner angles, mentioned in the postulate, the greater the outer and the less the inner angle between the considered lines. Apparently, the inner angles on the segment at the side of convergence, i.e., where their sum is smaller than $180^{\circ}$, are segments of the outer angle at the apex. The proof of this assertion will be provided following the proof of the fifth 
postulate. Meanwhile it may be mentioned that if this assertion is true, the dependence between the angles at the segment and the angle at the apex between the considered straight lines becomes clear.
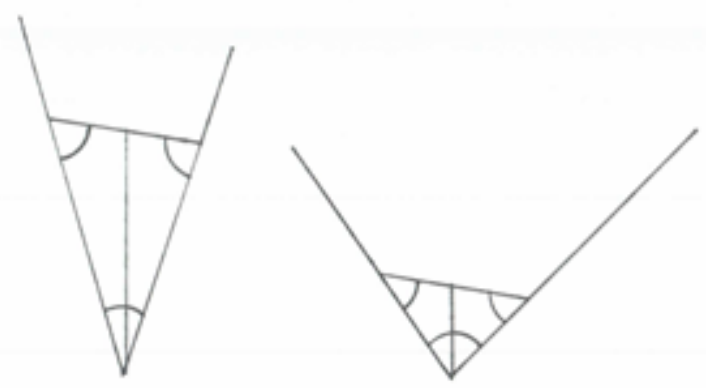

Figure 4. Relation of angles and distances of a segment from the apex of angle between straight lines

If one builds on two straight lines far from the point of their crossing angles, at the inner realm they will equal $180^{\circ}$, whereas the sum of outer and inner angles equals $360^{\circ}$, and will not define convergence or divergence of the straight lines considered. The introduction of the additional (defining) line permits to break the above angles into unequal parts, and unity of the defining line provides rigid dependence between the inner angles of the defined lines: the greater one of them (depending on the slope of the defining line), the lesser the other one (fig. 5).
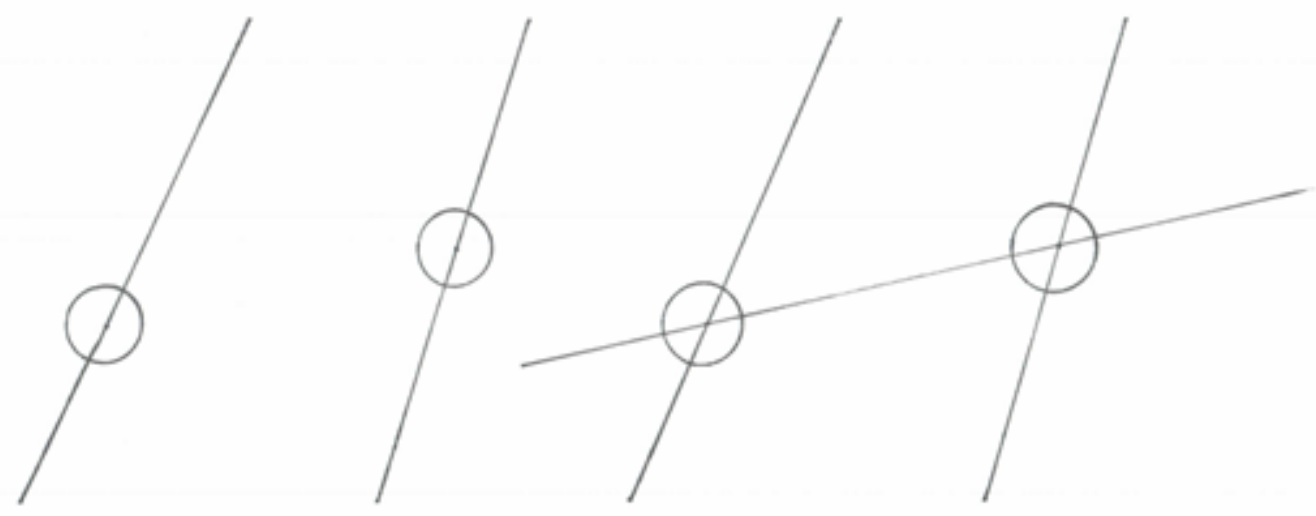

Figure 5. Angles without and with a defining line

In the interest of convenience, let us set the defining line parallel to the line of the shoulders. In that case, it lies in the dimension $\mathrm{x}$. The correspondent direction could be marked as $+\mathrm{x}$ and $-\mathrm{x}$, but, accepting a certain point for zero, we will find that one of the defined lin In case the defined line does not incline to the left, nor to the right, i.e., if by its elongation each subsequent segment will not be situated to the left nor to the right of the antecedent ones, we shall call such an angle straight $\left(90^{\circ}\right)$. If angles are found at the other side from the direction considered, the straight line declines from it, and the angle is obtuse $\left(>90^{\circ} ; 180^{\circ}<\right)$; and if during prolongation of the straight line, the segment moves towards the considered direction, then the line is inclined to said direction, and the angle is 
acute $\left(90^{\circ}<\right)$. es will go in the "positive" direction on the negative segment and/or, in contrast — in the "negative" direction on the positive segment (fig. 6).

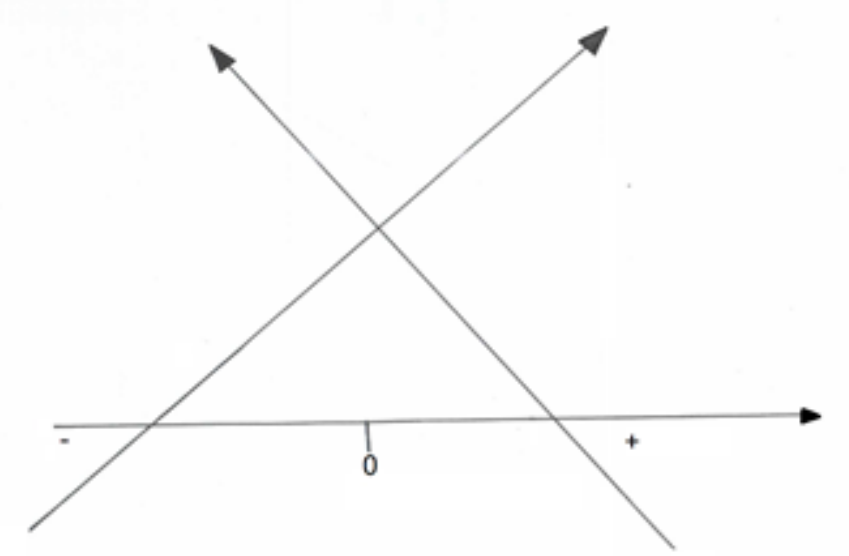

Figure 6. Accepting defined lines' directions towards the defining line for negative and positive

For this reason, it will be preferable to mark these directions as right and left. The greater the distance in the direction, set by the defining line, past the defined line per unit of its length, the stronger it is inclined in the given direction and declined in the opposite one. The dimension $\mathrm{x}$, containing the defined line, is represented by two directions, whereas the dimension $y$ is represented by only one direction, common to both defined lines - from the defining one.

If the sum of inner or outer angles at one side of the defining line equals $180^{\circ}$, then both angles may be straight, hence, the lines do not incline towards one another, or one of the angles is acute and the other is obtuse. In both cases, the sum of the inner angles of the defined lines becomes equal to that of the outer and inner angles of each from the defined lines. Hence, the inner angle of one of the defined lines equals the outer angle of the other defined line. Such angles are codirectional (in the considered orientation, right or left). Thus, by elongation by an equal length the straight lines pass the same distance in the direction, given by the defining line. For this reason, by their elongation at an equal length, the distance between them remains constant. In other words, the given straight lines are inclined in the same direction, situated in different places. Hence, they are parallel ${ }^{3}$ (fig. 7).

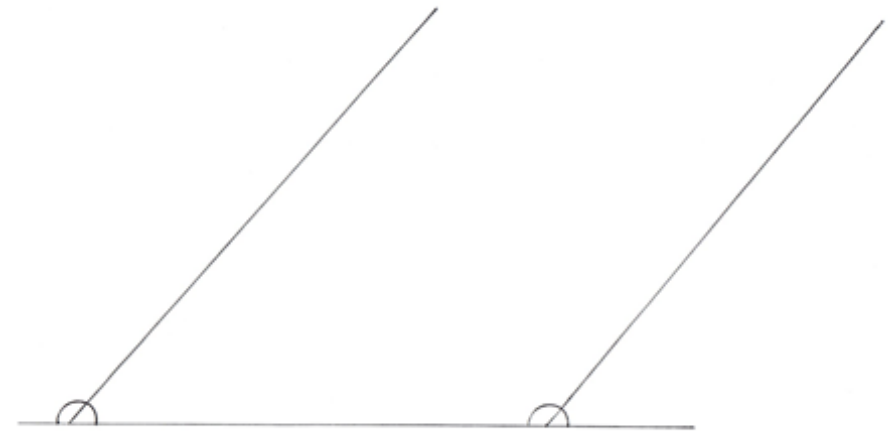

Figure 7. Parallel defined lines with angles at the defining line 
If the sum of the inner angles is lesser, (and the sum of outer - greater) than $180 \mathrm{o}$, then, at least one of the inner angles is acute. The other inner angle may be acute as well. In this case, both defined lines are directed towards the inner area (the right one leftwards; the left one rightwards) and they approach by their elongation (fig. 8).

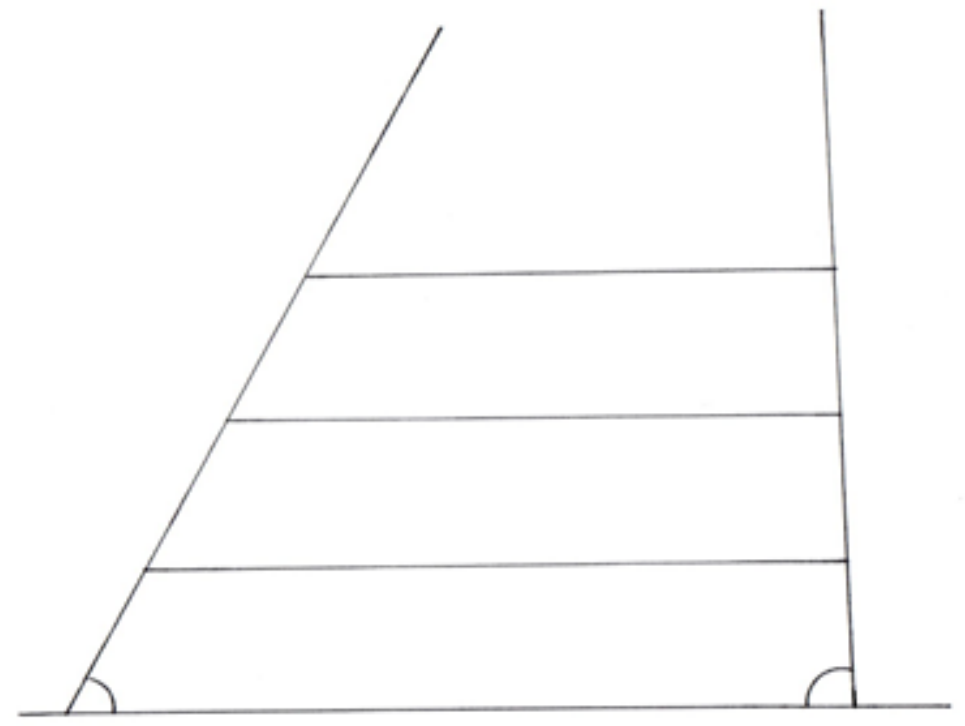

Figure 8 . The sum of the inner angles is less than $180 \mathrm{o}$, both angles are acute

If the inner angle of the second line is straight, than that line does not incline towards the inner area and does not decline from it, whereas the first line is inclined by the acute angle to the inner area, hence it approaches the second one (fig. 9).

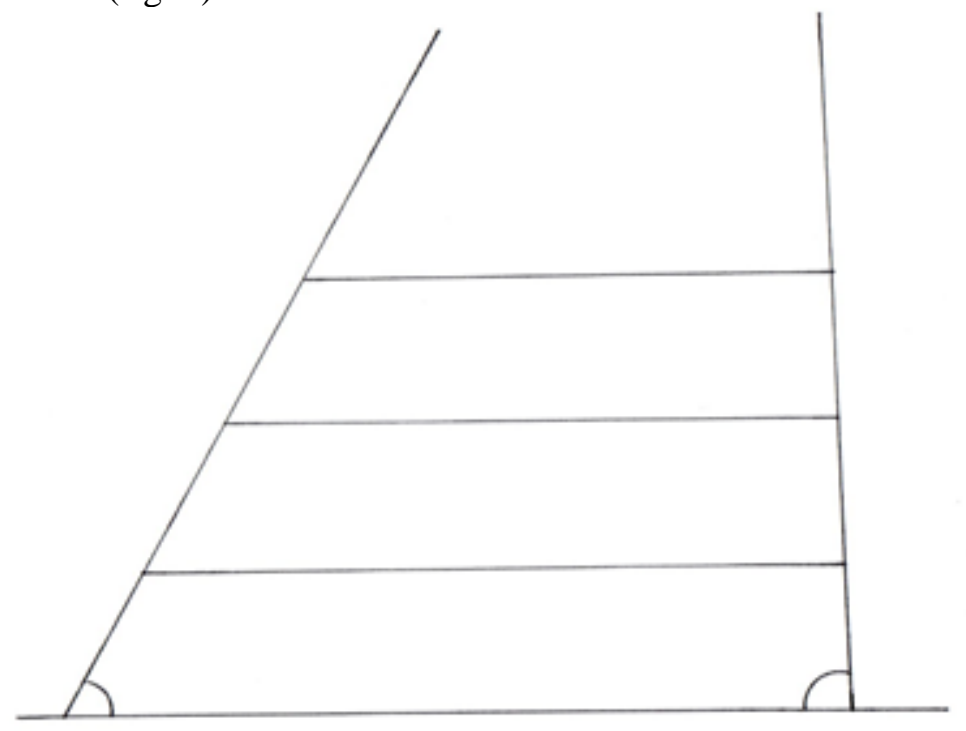

Figure 9. The sum of inner angles is less than 180o. The left angle is acute, the right angle is straight

Finally, the second angle may be obtuse. In that case, it is lesser than the outer angle of the other defined line. In that case, the latter passes towards the other line at a greater distance than the former one. As a result, by the elongation of these lines, the distance between them is reduced ${ }^{4}$ (fig. 10). 


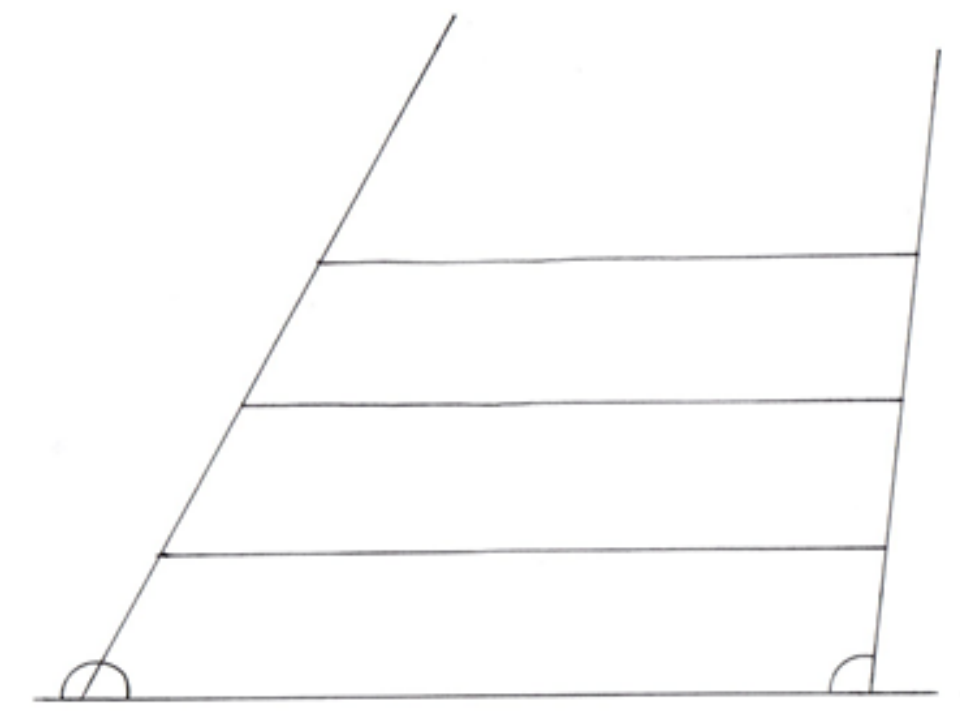

Figure 10. The sum of inner angles is less than 180o. The left angle is acute, the right angle is obtuse

As was shown earlier, if straight lines on a common plane approach one another, they should cross by their elongation.

On the other side of the defining line, where the sum of the inner angles is greater than 180 o the defined lines will diverge away from one another.

This is the proof of the fifth postulate in its original wording. Let proceed to the consequences.

The postulate may be proved also by the consideration of inner angles on both sides of the defining line.

The sum of all the angles equals 360o. If the sum of the inner angle on one side of the defining line equals $180 \mathrm{o}$, that on the other side is of the same value. Then the degrees of inclination on both sides of the defining lines are equal. If the sum of the inner angles on one side is less, than on the other side it is greater than $180 \mathrm{o}$; therefore, the lines approach where the sum of the angles is lesser and diverge if it is greater.

Such an approach is more illustrative, but, being generalizing, it does not show, what is the cause of the convergence or divergence of the defining lines.

Instead, with its help one other thing becomes clear: the sum of the angles on one side of the (defining) line does not equal the sum on its other side within the same angle. Hence, the angle does not equal itself (fig. 11).

The author considers that this is a demonstration of the dialectic principle of the unity and struggle of opposites in geometry, the most illustrative example of which is the unity of mutually-defined contrary directions in the same line. 


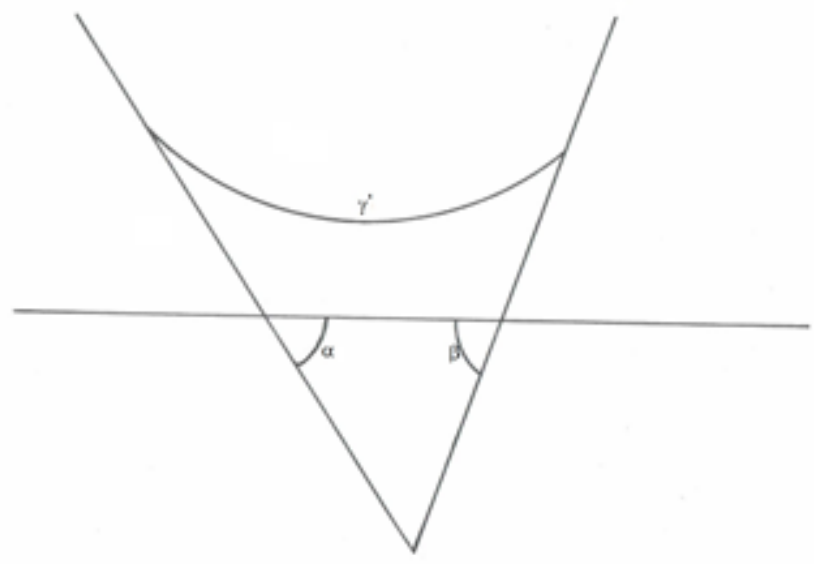

Figure 11. Construction of angle

The value of this angle should be equal to the sum of angles $\square$ and $\square$ minus the angle value of the line, on which they lean, i.e., $\gamma^{\prime}=\alpha+\beta-180^{\circ}$. Taking into account that $\alpha+\beta<180^{\circ}$, the considered angle is of a negative value.

Correspondingly, $\gamma=\alpha^{\prime}+\beta^{\prime}-180^{\circ}=360^{\circ}-\alpha-\beta-180^{\circ}=180^{\circ}-\alpha-\beta$ (fig. 12). Therefore, $\gamma^{\prime}=-\gamma$.

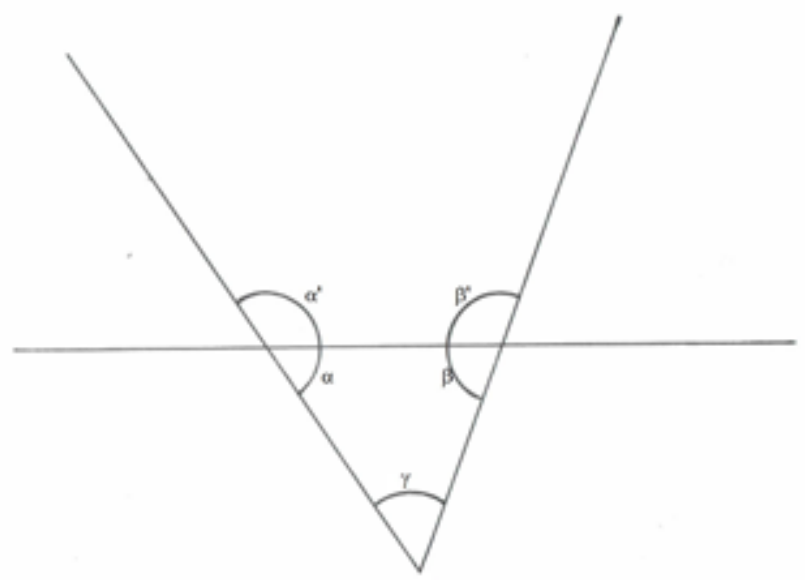

Figure 12. The totality of angles and anti-angles

Perhaps the shortest proof is that the sum of angles of a triangle equals $180^{\circ}$.

It should also be noted that, in contrast to particles and anti-particles, contraries exist in unity, like the poles of magnet (whose monopoles some physicists nevertheless attempt to find).

The defining line divides geometrical polarities: line convergence and divergence.

It is also necessary to note also that the absence of the inclination is marked as $0^{\circ}$, although the distance between parallel lines is constant and never tends to zero, in contrast to positive values of angles, meeting at zero of the first order, at the point of the apex. Such apparent pretense enables the finding of the additional negative angle ${ }^{5}$. In contrast to the afore-mentioned anti-angle, it is situated beyond the zero boundary not of the second (defining line) but of the first order, i.e., beyond the point of crossing. It 
differs from the positive angle by the direction of inclination, and also, by the quasi-turn of lines that is the changing of their position and the position of their sides. For greater visualization of this sequence, it is worthwhile to prove that the inner angles by the defining lines are segments of the outer angle of the defined lines.

Let us make a parallel translation of the defining line at the apex of the angle between the defined lines. Let us consider initially the side that is situated on the opposite side of the apex that was put on the same level with the latter.

The angles, mentioned in the initial wording of the postulate, equal the outer angles on the other side of the defining line. This follows from the fact that the sum of the values of the outer angle with the adjacent inner one of the same side or the outer one of the opposite side equals $180^{\circ}$.

According to the parallel translation, the outer angles on the considered side will provide segments of the outer angle between the defined lines, whose sum with the inner angle equals $180^{\circ}$, whereas the sum of the outer and inner angles, situated on the one side of the defining line, remote from the apex, equals $360^{\circ}$. The difference should be attributed to the corresponding inner angles, which, merging together, lose support on the straight line that forms $180^{\circ}$ (fig. 13).

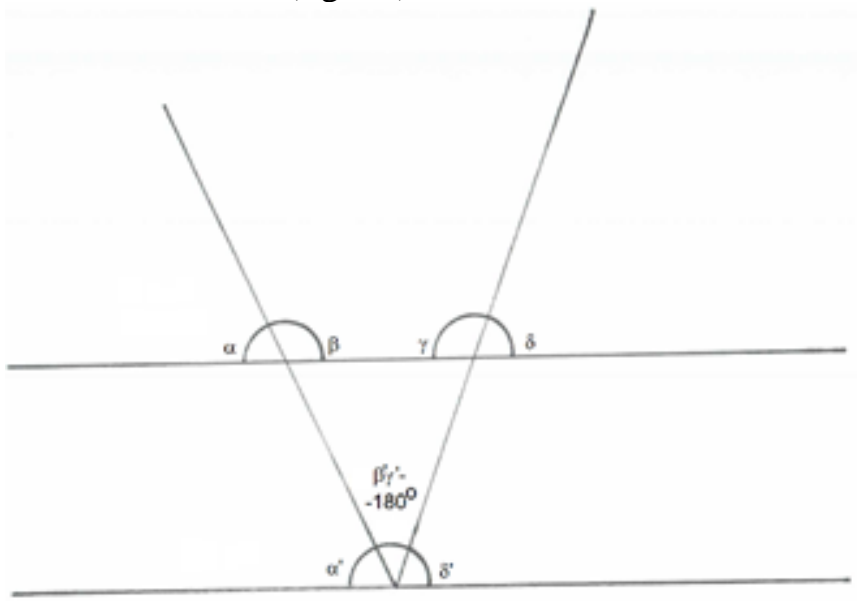

Figure 13. Parallel translation of angles

Beyond the point of crossing the defined lines change both interposition and direction. Their inner sides become outer, and vice-versa. The same applies to the inner angles on the side of the apex after the parallel translation of the defining line. During this process they exchange places, remaining alternate angles relative to the outer angles of the opposite side. The former outer angles, whose initial sum exceeded 180o, move inside. Now, like the inner angles on the other side of the defining line, they merge and lose these degrees together with the straight line.

Such "rotation" of lines or angles exists only through the overall consideration thereof (fig. 14). 

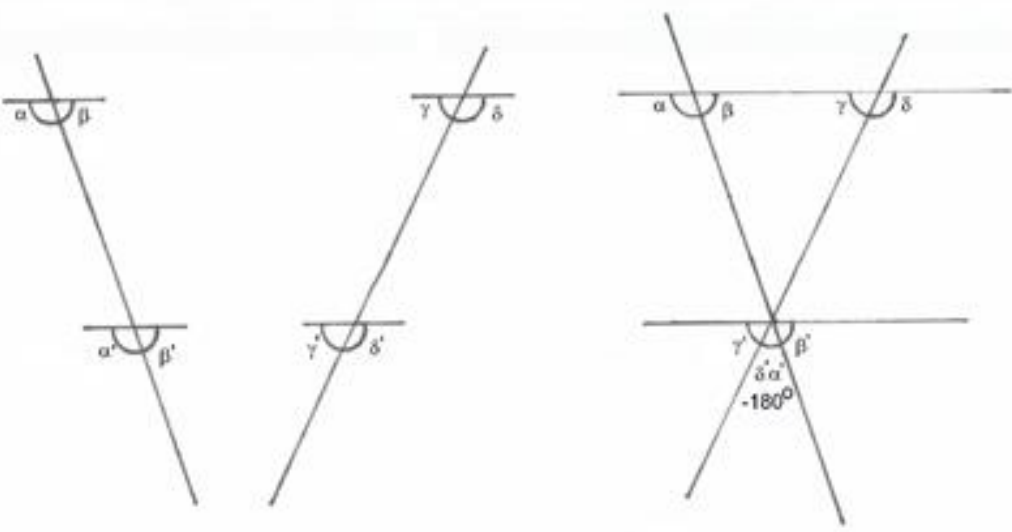

Figure 14. Quasi-turn of angles

Thus, inner angles by the defining line on the side of convergence are in fact the segments of the outer angle between the defined lines.

The given consequence introduces a correction to the principle of relativity in physics.

It is opined that the state of inertial motion is characterized by a constant speed and straightforwardness, defined by the motion relative to bodies, found in the same state (Physical Encyclopedic Dictionary, 1983, p. 602). However, if the speed of such a motion is constant, then after time equal to $\frac{S}{v}$ (or minus $\frac{S}{v}$ for the past), the distance between the objects should turn into zero. If this result is not the outcome, then the speed is alternating. This is the objects' motion along parallel trajectories, and also by skew or by crossed trajectories in the case of non-simultaneous passage of the crossing point. The greater the speed, the closer the length of trajectory to the line connecting objects, and the greater the distance of objects from the points of their opposition. Also, the displacement here is quasi-rotated, not accompanied by a centrifugal force.

\section{Thus, when non-Euclidean geometries represent geometrical relativism, then the geometrical groundings of the principle of relativity follow from the fifth postulate ${ }^{6}$.}

Since one dimension defines the distance between lines, whereas the other - the direction of convergence and divergence, the oblique lines, found on the same plane will cross by their indefinite elongation. In the three-dimensional totality, the lines during their elongation in the direction of convergence may not cross, and appear to be skewed.

Let us consider the applicability of the fifth postulate to this case as well. First and foremost, it should be noted, that there are no two sides; rather, at least two pairs. They may be found when the defining line is connected with each defined line by means of a common plane.

If the angle between the sides is greater than $90^{\circ}$, the sides decline from one another. In the extreme case, when the angle between the sides equals $180^{\circ}$, we obtain a plane with defined lines, coming from the opposite sides of the defining line. It is clear that in this case the defining line may only diverge.

Nevertheless, taking into account the equality of mutually negative angles, we may predict on which side the convergence will occur. Let us take the inner angle of one defined line and the outer angle of the other line. The latter equals the inner angle of the same line on the other side of the defining line. If the sum of these angles is less than $180^{\circ}$, convergence proceeds on the side adjacent to the inner angle and opposite the outer one; if it is greater - vice versa (fig. 15). 
The case under consideration reveals a new aspect of the fifth postulate. Namely, the sum of the counterdirected, but not of the co-directed, angles, may be the criterion for the convergence/divergence of the defined lines.

The generalizing rule of the difference of the sum of angles from $180^{\circ}$ is applied only to counter-directed angles; whereas for co-directed angles - simple inequality or equality by concretization of their positions. Thus, returning to a single plane, the sum of counter-directed angles at parallel defined lines should be equal to $180^{\circ}$, whereas their co-directed angles should just be equal to one another, whereas their sum may vary from $0^{\circ}$ to $180^{\circ}$, excluding the limits. As to unequal co-directed angles, the result is dependent on their arrangement. Thus, if the right angle at the left line is smaller than the right angle at the right line, then those lines approach, not vice versa; that would be true for counter-directed angles, since the result depends only on their sum. According to the fifth postulate, if the sum of the internal angles, i.e. the left and the right angles, is less than $180^{\circ}$, then the lines approach.

The angles between the sides of the skew lines may be straight. In that case uncertainty may appear. Thus, if one of the defined lines is perpendicular to the defining one, and the other is not, it is not clear to which side, containing the perpendicular line, should the corresponding part of the other skew line incline (fig. 16). The same problem appears for other values of angles, i.e., $60^{\circ}$ at one portion of one defined line and $110^{\circ}$ and $70^{\circ}$ at the sides of the other skew line.

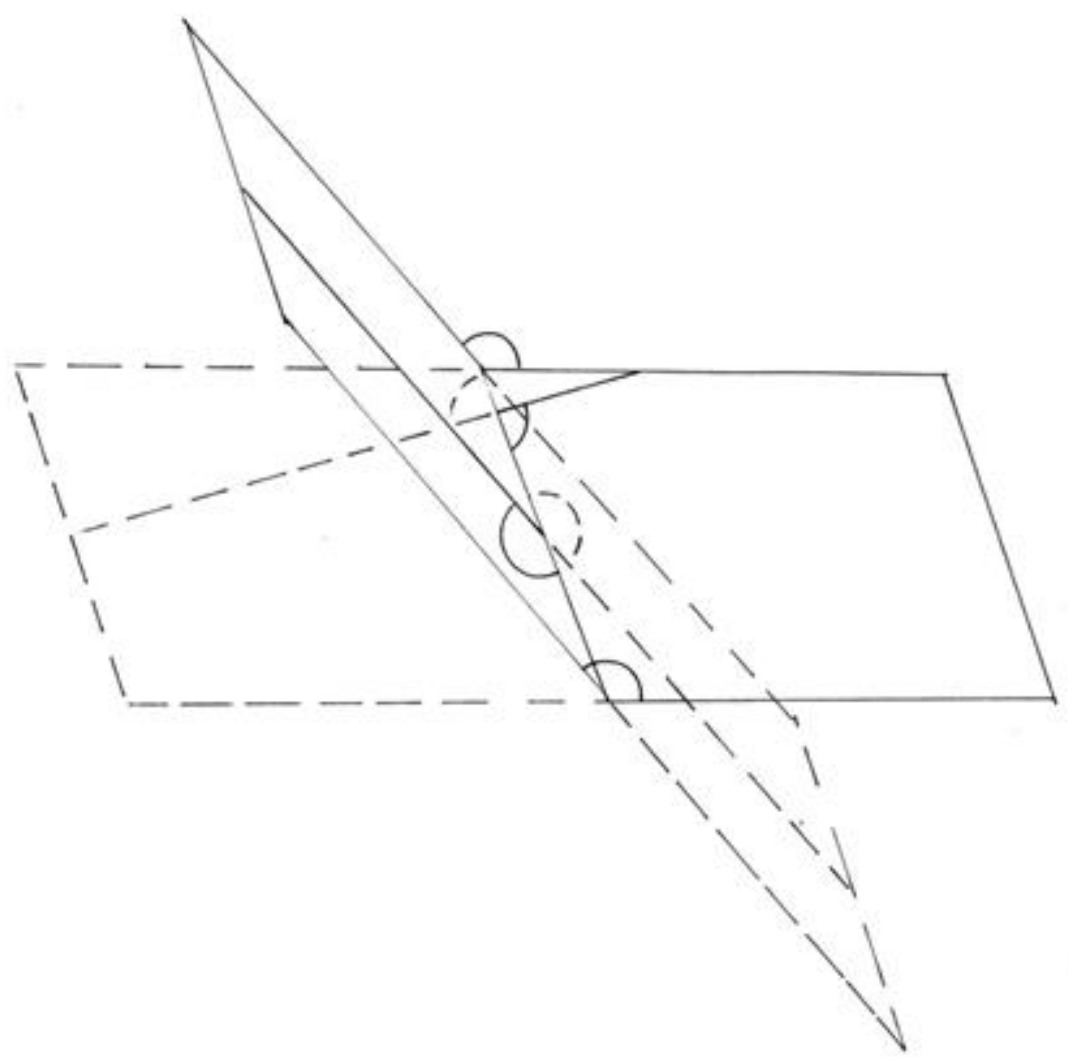

Figure. 15. Obtuse angle between the sides containing the defined skew lines 


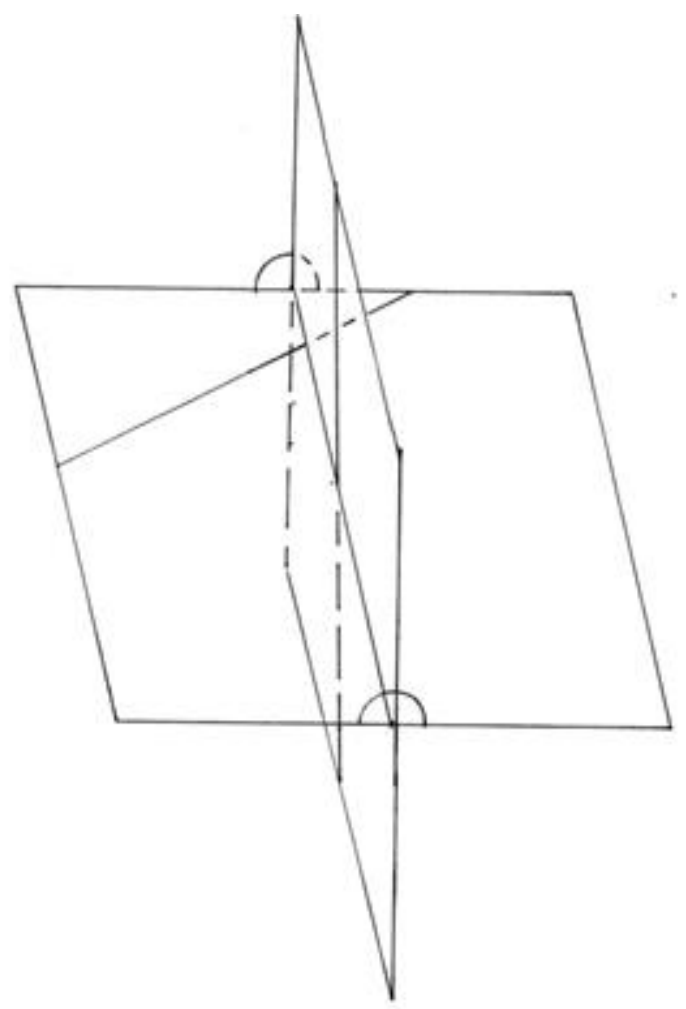

Figure 16. A straight angle between the sides containing skew lines

Then the new defined line should be drawn in such a way that the angle between the sides, containing defined skew lines, will differ from 90o.

If the angle between the sides is less than 90o, and the sum of inner angles is less than $180 \mathrm{o}$, the defined lines approach, since the projection of each of them on the other plane, behaves in correspondence with the fifth postulate (fig. 17).

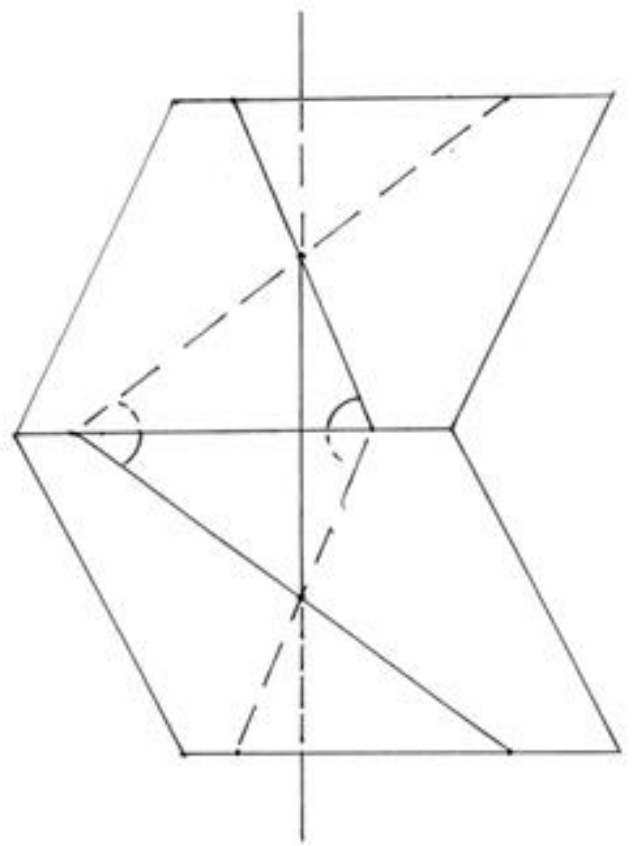

Figure 17. Acute angle between the sides containing skew lines 
Another issue is whether the points of crossing lines with projections correspond to the opposite points of the lines. The answer is negative, since the line, crossing opposite points, should connect the skew line by the shortest distance, i.e. to be perpendicular to both lines. This is impossible, if it crosses a plane, which, in turn, is crossed by the defining line. Of course, it is possible to find such a line by the trial and error "method", but the clear criterion for finding the points of opposition is missed here. Thus, the fifth postulate in its canonic form, whereby two defined lines crossing the same defining line, cannot be applied to skew lines in an effective way.

If the angle between the sides is less than $90 \mathrm{o}$, and the sum of the corresponding angles equals $180 \mathrm{o}$, then each of the projections is parallel to the defined line lying on the same plane. For this reason, the approach or removal of the defined lines cannot be defined by their projections, as in the previous case. Instead, through each defined line and its projection on the plane, parallel to same, containing the other line and its projection, may pass. Taking into account that the directions of the approach and removal are unknown, the defined lines and their projections should be shown completely, by both sides of the defining line, i.e. by a cross. Each of the defined lines crosses its projection and defining line at the point, that for brevity I will call the point of crossing.

If the defining line is perpendicular to both defined lines, it connects the points of their opposition — and, strictly speaking, is not the defining line according to the postulate.

Let us consider the case, in which the angles between defined and defining lines differ from straight ones, and the perpendiculars to the parallel planes connect one of the defined lines and the projection, which is parallel to it. The latter in this case is both the orthogonal projection of the given defined line on the other plane and the projection of the other defined line.

If from the point of crossing, lying on the first defined lines perpendicular A will be drawn on the parallel plane, it will connect skewing projections of the defined lines (wholly depicted as traits) at the points of their opposition.

If from the point of crossing, lying on the correspondent orthogonal projection of the defined line, one will draw perpendicular B on the parallel plane, the former will connect the skewing defined lines (whose open parts are depicted as a continuous line, and the hidden - by traits) at the points of their opposition (fig. $18)$.

If the perpendicular, connecting a defined line with parallel planes cannot reach the projection, it cannot connect the points of opposition, since it will pass beyond the corresponding line. Here, as in the case when the sum of inner angles is lesser than $180^{\circ}$, and the angle between the sides is lesser than $90^{\circ}$, the points of opposition may be found by the trial and error "method" only.

An improved drawing follows from the aforementioned options. Namely, when one of the skewed lines is on each of the parallel planes, the orthogonal projection of the other line and a defined line, which connects a defined line with the projection of the other defined line, and which may be chosen independently to the position of the other defined line, situated on the parallel plane, then the approaching or removal of the skew line may be judged by the sum of the inner angles, in accordance with the fifth postulate, that applies to each of the planes. In such a case, the points of crossing of the defined lines with the orthogonal projection of the other defined line, are the points of the opposition of the skew defined line, as well as their projections. 


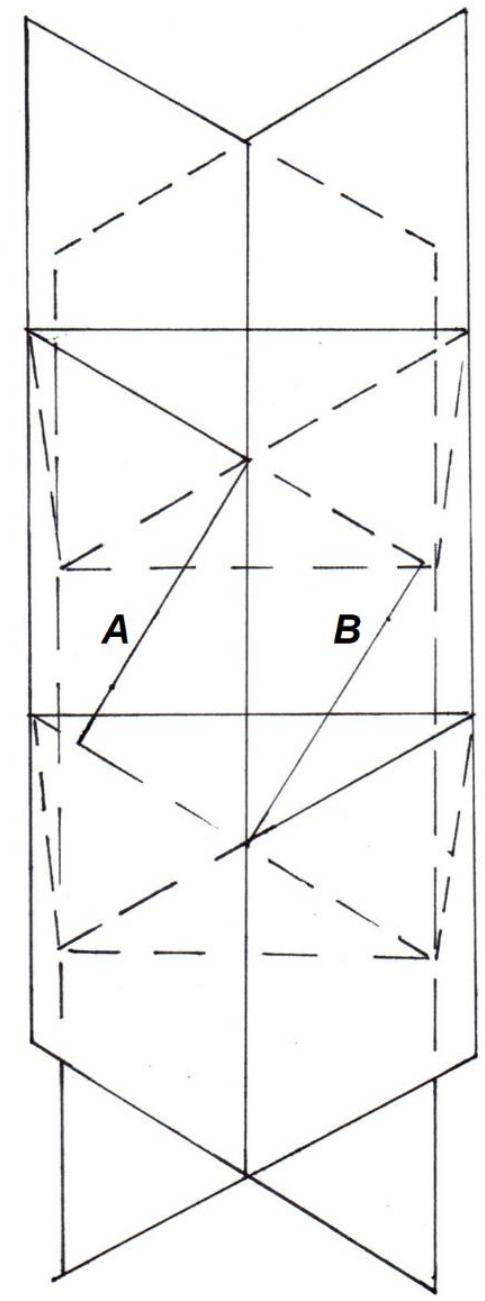

Figure 18. Points of opposition of skew lines. The angle between the sides is lesser than $90^{\circ}$. The sum of inner angles equals $180^{\circ}$. The defining line is not perpendicular to the defined ones. One line and its projection on a parallel plane may be connected by lines that are perpendicular both to them and to parallel planes

Along with the complication of proof of the fifth postulate, a simplification of the original wording took place. Yet Nassir al-Din Tusi introduced the axiom of parallels, according to which only one straight line passes through a point, parallel to the given one. Though the book of this mathematician was published in 1594 in Rome, the said axiom was accepted only when it was re-discovered by Playfair 203 years afterwards.

As in the case with the initial wording, there were unsuccessful attempts to prove it by means of Euclid's first four axioms. Lobachevsky, proceeding there from, assumed that an infinite number of straight lines pass through one point, parallel to the given (Lobachevsky, 1946, p. 82 - 86). The said conclusions seem incompatible, but the author considers that they are rather antinomous.

Let us proceed to proof. According to Euclid, "Parallel straight lines are straight lines which, being on the same plane and being produced indefinitely in either direction, do not meet one another in either direction." (Euclyd, Essentials, p. 14) Such a criterion is idealistic, since it cannot be applied in daily practice, from which it was derived. As follows from the initial statement, such a sequence is true only in the case, that the lines, belonging to the same plane, proceed at a constant distance from one another ${ }^{7}$. Just 
this criterion is used in practice, the evidence of which is the name "parallel", deriving from " $\pi \alpha \rho \alpha \alpha \lambda \lambda \eta \lambda \circ \varsigma$ ", that is "near one another".

Since the sums of the constant values are constant, if lines are parallel to the given one, they are parallel to one another. Inter alia, if they pass through a common point, the distance between them after the point should be the same, i.e., zero. Hence, a unique line passes through the point, parallel to the given line. Euclid defines a line as "length without width" (Euclyd, Essentials, p. 11). Nevertheless, no geometer applies the term "pseudo-lines" to drawn lines, although they have a negligibly small width. The latter may be split along a cluster of parallel lines in accordance with Lobachevsky.

Let us note: the passing of unity into a multitude here causes antinomies. Such an aspect will be elucidated in the following paragraphs. The author came to this theme during polemics with the basic statements of physics of the XX century. It is enough to note that the images of particles are formed by the same particles or even more coarse bodies (water molecules in the chamber of Wilson), in the absence of focusing, in contrast to a microscope; that the relation of an electron's image to its original in the Wilson chamber is one thousand times greater than that of the earth to man (in the order of $10^{10}$ and $10^{7}$, correspondingly). In order to understand that, as they approached the abyss, physicists accepted as a bolster the so-called elementary particles, whose elementariness is doubtful. At the same time, by capturing the micro-world, they solved philosophic tasks. Unfortunately, the idea, whose need appears, requires time for its elaboration, which may last for hundreds and thousands of years. The author believes that "light" for Einstein is the same thing as the "water" placed in the base of the world for ancient Greek philosopher Thales. Such misapprehensions are very clear to common people, because scientific elucidation does not belittle the depth of the questions posed to those thinkers. Further, the author will mention the main disagreements with non-Euclidean geometry in order to show what the main contradiction is between non-Euclidian geometry and the basic structure of the so-called space.

\section{INTERNAL INFINITY, HISTORY AND REALITY}

In mathematicsin general, and in geometry in particular, the notions of finite and infinite values are key. Thus, it may be noted that "Every finite group $\mathrm{G}$ can be realized as the group of self-homotopy equivalences of infinitely many $<\ldots>$ rational elliptic spaces X" (Costoya, \& Viruel, 2014). I think that some key notions require further philosophic elaboration, which sometimes may affect correspondent theories.

I will begin the consideration from the modern view of the appearance of the Universe. Currently the Big Bang theory is accepted as being its beginning.

Nevertheless, physical devices have their limitations.

The $17^{\text {th }}$ century philosopher, Gassendi, distinguished between the world that is the visible part of the Universe and the Universe itself (Gassendi, 1956, p. 187). Although the world that is observed by modern astronomers is broader than that which could be seen by Gassendi and his comrade Galileo Galilei, in both cases what is observed is the world, not the entire Universe.

All that we really know about the Universe is that it is united. As will be shown later, there is no void. Therefore, the said unity is not formal but physical. At the same time the Universe is composed of galaxies, stars, planets, humanity, etc. Hence the question follows: why does it not represent the only existing basic unit - the indivisible bloc of being?

The explanation perhaps is that the being is characterized by greatness. This implies that it should be greater than anything existing inside it - and this lesser part should be separated in reality, i.e. physically. 
Because any part of the being is great in itself, it should be greater than its own parts. Hence, both the Universe and its subdivisions are infinite.

Infinity means that any unit is composed of a multitude, and any multitude — of units. Constant struggle between them, i.e. movement, proceeds. The reasoning thereof may lead to a unilateral conclusion regarding the existence of irreconcilable contradictions, antinomies revealed by Kant (Kant, Writings in 6 volumes. Vol. 3, $404-405 ; 410-411 ; 418-419 ; 424-425)$. Their portrayal was shown earlier in the examination of the axiom of parallel lines. The same is true for the paradox of Democritus (see below).

Since the struggle between unity and multitude permeates the entire being, the amount of movement per unit of matter is constant. The same amount of changes occurs in the course of one second in a crystal, as in the equal volume of smoke. The crystal is distinguished from the smoke by less unordered, therefore, greater ordered, motion. It is also more or less autonomous from its surroundings. Therefore, such motion is relatively closed, and, taking into account its motion within the Solar system, Earth, even as a crystal in a man's hand, moving along with him, is absolutely open. Hence, the crystal is an accumulation of streams, whose speed provides its hardness.

Some physicists of the second half of the 19th century came to the latter conclusion (Maxwell, 1954, pp. 11, 103, 131); nevertheless, they did not notice that these streams did not simply flow, like a river does but, similar to the flowing of cytoplasm and blood, they are focused on the formation of a perfect and constant shape, which is so constant that we perceive it as completely immobile.

Let us note that we do not see the crystal itself, but rather its image, that is formed by the constant flow of light, flying with tremendous speed, by the motion of eyes, by means of the photochemical processes in the retina and by the uninterrupted impulses in the brain. So, here as well, the apparent immobility of an image is a display of its constancy. The level at which the constancy of an object is displayed, can be called "the significant level".

In fact, the crystal is a kind of a living organism, and its tendency to preserve its constancy is a vestigial kind of instinct of self-preservation. From the mathematical standpoint, it is important that the significant level is finite despite its infinite groundings.

Then, the difference between finite and infinite values goes beyond the frame of mathematics and belongs to metaphysics.

The conclusion about the infinite nature of any being, contradicts the notion of infinity as a number, greater than any other, which is implicit.

Thus, the atomists of ancient India argued that "The hypothesis about infinite divisibility...is clearly absurd, since it contradicts certain immediately considered facts. One cannot debate which is greater by value - a high mountain or a small grain. Nevertheless, according to this hypothesis, a mountain may be divided into an infinite number of parts, i.e. this implies that a mountain consists of an infinite number of parts. But the same could be said about a grain. Hence, both mountain and grain consist of infinite amounts of parts, and therefore, they may be considered to be equal in size." (Chattopadhyaya, 1981, p. 292).

That is the antithesis of infinite divisibility. The synthesis of the theoretical statement about infinite divisibility and visible differences in the size of objects is that infinity is not the greatest amount, but the ability of any component to be composed. 
In this case, any objects, with which we perform arithmetic operations, are embodied infinities, and we can judge the infinity by their properties.

Some thinkers approached such a notion.

Thus, when Mersenne remarked "One infinity cannot be greater than the other one", Descartes answered: "So why not? What is absurd here?" (Descartes, p. 589).

In his letter to Dr. Bentley, Newton unequivocally affirmed that "The infinite number of those parts in a foot is not equal to, but twelve times bigger than the infinite number of them in an inch" (Newton, p. 17). Nevertheless, in physics Newton adhered to the atomistic concept: "These primitive Particles being Solids $<\ldots>$ so very hard, as never to wear no break in pieces; no ordinary Power being able to divide what God himself made one in the first Creation" (Newton, p. 400).

The peculiar properties of infinity, perceived as the totality of all numbers, Galileo Galilei based on the paradox of square numbers: "The amount of the square numbers decreases uninterruptedly and in very great proportions, when we come to greater numbers, thus from the numbers up to one hundred the amount of square numbers is ten, i.e., one-tenth part; in the amount up to ten thousand, that of square numbers will be one hundredth part only; up to one million - one thousandth part only" (Galilei, p. 140). On the other hand, "The amount of the square numbers is the same as that of roots, since each square number has its root and its root - its square; no one square can have more than one root and no one root more than one square number. Therefore, the amount of the square numbers equals that of all the numbers, since there is the same amount of roots, and all the numbers are roots." (Galilei, p. 141). Hence, "The properties of equality and also of lesser and greater value have no place, where the deal about infinity goes, and are applied to finite amounts only." (Ibid., p. $140-141$ ).

The great scientist, like many thinkers, was so fascinated by the paradox, which corresponded to his views, that he did not bring the solution all the way to its conclusion. Not one root corresponds to a square number (excluding one), but multiple roots; and the greater the root, the greater its multiplicity factor, equal in this degree to the number itself: in a unit, one unit is contained; in four - two twains; in nine three terns; in sixteen - four fours etc. Thus, by the increase of the number row, the amount of roots, accounting for a one square number, increases in arithmetical progression.

Cantor illustrated a similar conclusion in his theory of sets, which is currently recognized. According to him, between simple infinite sets (i.e., between sets of all natural and all even natural numbers) there exists a one-to one correspondence. In this case, number 2 from the second set corresponds to number 1 from the first set; number 4 - to number 2; number 6 - to number 3 ; number 8 - to number 4 , etc. "to infinity" (Courant, \& Robbins, 2001, p. 105). Such a conclusion seems veracious to those who recognize the peculiar properties of infinity, and at the very least dubious to those who deny them.

Let us note that we cannot sense infinity immediately. Its peculiar properties should be shown in the tendency of a row increase, as Galileo did. In contrast, Cantor shows not the process, but the finished result in the example of one pair of finite sets, in the given case, $\{1,2,3,4\}$ and $\{2,4,6,8\}$.

Each element of the first set here is two times lesser than the correspondent element of the second set. Since $2 \frac{n}{2}=n$, the element of the second set, corresponding to the half of the first one, equals the latter element of the first set. In the given case, 4 from the second set is positioned against 2 from the first set. The following two elements of the second set (6 and 8) go beyond the boundaries of the first set, limited here by the number 4 . If the number of elements is odd, e. g., 3, then the number from the second set, falling on the middle (4) is greater than the latternumber of the first set by one unit. Therefore, here more 
than half of the elements of the second set go beyond the first one and, in contrast to Cantor's assumption, cannot be part of same.

Cantor also came to conclusion that an infinite set, composed of subsets of any infinite set, is greater than the latter (Courant, \& Robbins, 2001, p. 111 - 122). I consider the conclusion concerning the inequality of infinities as a progressive step; nevertheless, the method of demonstration, in my view, is mystical. Let us consider two "finite" sets: on the one hand, the set $\{1,2,3\}$; on the other hand, a set composed of its subsets: $\{\varnothing,\{1,2,3\},\{1\},\{2\},\{3\},\{1,2\},\{1,3\},\{2,3\}\}$. It can be seen that the unit from the second subset is identical to those from the third, sixth and seventh subsets. I.e. one real unit corresponds to the four on record. The same applies to the other elements with the exception of the empty set. This conclusion may be tested empirically, employing three matches. We may group them in different subsets, and the set, comprised of said subsets, will be the totality of all combinations (i.e., the total time), but in each combination the amount of matches will be not greater than three. I.e., a one-to-one correspondence exists between the set, composed of subsets of the given set and the given set. It may be seen, however, that curly brackets have a real physical meaning. There are unequal distances between matches, boxes of matches, etc. Taking those objects into account, the absence of a one-to-one correspondence may be concluded.

Such exercises by mathematicians caused mistrust among physicists towards infinity. Hawking writes: "Because mathematics cannot really handle infinite numbers, this means that the general theory of relativity... predicts that there is a point in the universe where the theory itself breaks down" (Hawking \& Stephen, 1988, p. 46). Thus, relying on the concepts of his predecessors, Hawking accepted even their faults as signs from above.

In the case of the infinite divisibility of a value, the limit of its division is by no means infinity, rather the infinite totality of its, and only its, parts. Dividing such a value physically, we would obtain the given volume of the absolutely amorphous matter, and we wouldn't know, how to study it, since any significant level, including that of physical apparatuses, is finite. We can do otherwise, accepting infinite for the definite amount. In this case,

$$
\lim \mathrm{x}: \infty=\mathrm{x}: \infty_{\mathrm{x} \neq \mathrm{y}}=\mathrm{x}: \mathrm{x}=1_{\mathrm{e}}
$$

where $1_{\mathrm{e}}$ is elementary, infinitesimal or an infinitely small unit, indivisible further.

\section{METAMETRY}

There have been attempts to conclude the properties of space from the metaphysical standpoint. Engels ironically writes about such views, seemingly, "The first line appeared as result of a dot's motion in space, the first surface - from the motion of the line, the first body - from the movement of the surface, etc." (Marx \& Engels, p. 39).

Currently the main approach to the problem is based on the notion of higher mathematics; as well as some physical disciplines, e.g., electrodynamics (Nazarov, Treil \& Volberg, 2003). Though some notions may proceed in the right direction, nevertheless, I can conclude that the solution is simpler.

It may be compared with digging a pit for a building that does not require any elaborate knowledge, such as plastic anatomy for sculptures. Nevertheless, it is quite important, and furthermore, it was never dug before. It is grounded both on the real notion of infinity and the ideal of the infinitely small unit. 
For the existence of infinity, the function, determining the co-existence and not the coincidence of its components is required. This function is direction, one at least. Let us then add infinitely small units to a given one. As a result, we would obtain two contrary directions. The point is that co-existence is mutual. Not only the considered unit co-exists with the added one, but also the added ones with the first one. Hence, the totality of two mutually defined contrary directions or dimension.

The unit in unidimensional totality represents a segment with an elementary length. Zero boundaries between the neighboring units are dots. In order to move from the infinitely small to the infinitely composed level, it is necessary to replace zero boundaries on self-identical transitional regions.

Aristotle indicated: "Plato unequivocally objected to the recognition of a dot as a kind of genus, considering this to be a geometrical fiction; the beginnings of the line he often called "indivisible lines". However, it is necessary that [these] lines should have a limit. For this reason, where a line exists, a dot exists." (Collected Works in 4 volumes, Vol. 1). Later, the statement according which lines consists of dots, was criticized by such philosophers as Boetius, Ibn Sina, European scholars, Spinoza, Bayle.

Such critique permits us to resolve the geometrical paradox, claiming that the amounts of dots on both small and long segments are equal.

Let us draw from a dot, that is found opposite the middles of small and large segments, lines which connect them. Each line will mark a pair of dots on both the small and the large segment. When the lines are drawn the total amount of dots will be equal (Courant \& Robbins, 2001, p. 110) (fig. 19)

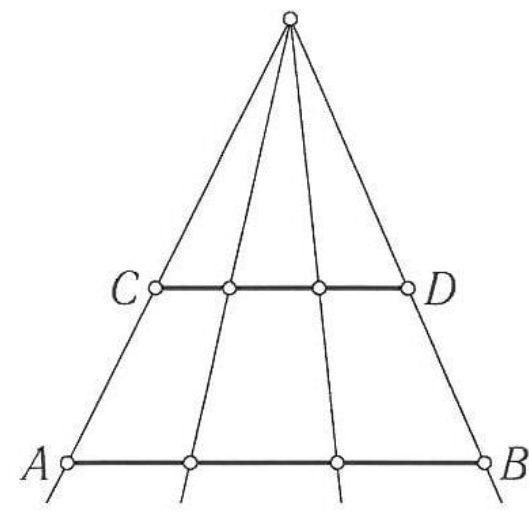

Figure 19. Demonstration of one-to-one correspondence of dots of two segments

We deal here with a notional illusion, resulting from the ignorance of two dimensions. Thus, lines drawn to the segments are considered as unidimensional ones, though on the drawing they possess a very small second dimension, i.e. width, say $0.1 \mathrm{~mm}$ for an acutely sharpened pencil. Such a line will mark not dots, but the tiniest segments. The smallest, equal to the width of a line, will be segments of the perpendicular, and with the angle diminishing, the length of such a segment will grow. It is natural that size of such a segment is greater in the large segment and vice versa.

One can use lines without width. Then the drawing should be dissected. In this case, the segment, found between slits, will be longer in the larger segment.

When the drawn "dots" fill the greater segment, on the lesser one a third dimension will come into effect. Namely, some lines will lie on others. The length of the segment, lying on the other, which touches the smaller segment, will be less than in the larger one. 
The real values (the so-called bodies) are separated via movement. The moving unit would divide unidimensional totality on the rear and fore sub-totalities. It cannot enter the fore unit; and it cannot shift the entire totality of units. In order to allow the considered unit to pass, the fore one should move aside. Hence, the second dimension. The unit in the second dimension represents a square with zero boundaries of two orders.

It is remarkable that the infinitely small unit that had a prototype, the mathematical indivisibles, was rejected at the end of $17^{\text {th }}$ century. The use of indivisibles caused difficulties, first and foremost, in geometry. Thus, Kepler did not succeed in expressing the sectors of a circle in indivisibles (Kepler, p. 91). Cavalieri used indivisibles to calculate the volume of a pyramid. In order to avoid grave errors, he was forced to apply different methods (Ibid.).

The other method was the expression of a curve as a sum of small tangent and sub-tangent lines. Gradually it led to integral and differential calculus, found independently by Leibniz and Newton. It is remarkable that the founders were forced to exclude "infinity small values" of a lesser order. Thus, if $y=$ $x^{2}$, then $y+d y=(x+d x)^{2}=x^{2}+2 x d x+d x^{2}$. Since $d x^{2}$ is "in infinite times" smaller than the infinitely small $d x$, the former is neglected, and we conclude that $y+d y=x^{2}+2 x d x$. Hence, $d y=2 x d x$. The idea of calculus was even received as non-religious superstition and criticized by the outstanding subjective philosopher Bishop Berkeley (Writings, The Analyst: a Discourse addressed to an Infidel Mathematician, p. $395-442)$.

It is clear that the calculus is only an approximate operation, applied not to true infinity, but rather, to a countless amount. The most prominent example is the attitude of physicists, who use calculus, but reject infinity as the property of reality.

Trying to express an angle in infinitely small units, the author of the present article found that it represents by itself a two-dimensional totality. The lines composing it should, therefore, possess the second dimension or be unidimensional boundaries of two-dimensional bases. Accepting the width of a line for an infinitely small unit, we will find that it is dissected in the apex. This means the exaggeration of the internal content, hence, the absence of curves and oblique lines, hence, curved space on the basic level (fig. 20).

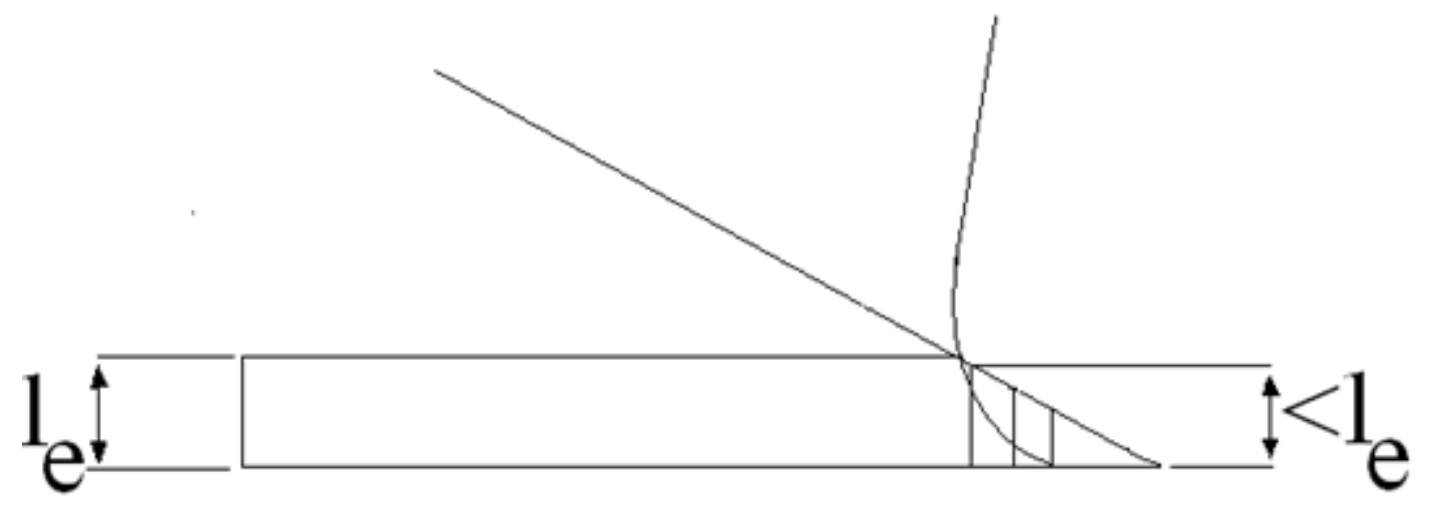

Figure 20. The section of the line composing the angle

The observed bending of light near stars is the result of gradual diffraction. Such a phenomenon with its corresponding explanation was predicted by Newton, in cases in which there is no void (Newton, p. 265). The same conclusion regarding curved and oblique lines emerges from the paradox of Democritus. Let us place the cone section parallel to the base. Are its surfaces equal to one another? If they are equal, then the parts, that are close to the base, are no different from those close to the apex, thus, forming a cylinder. If 
they are not equal, the cone cannot be created anew. Instead of a smooth side surface, a step would appear (Democritus, p. 240).

Democritus proposes a choice between two contraries. In effect, both of them are true: there are many steps, and each of them, as analysis indicates, is cylindrical. Nevertheless, the synthesis is not enough. Applying the same reasoning to longitudinal sections, one will receive a totality of cubes, in which volume is measured.

If for the acceptance of a curved line for a straight one man should convince himself of the truth of nonEuclidean geometry, then we naturally obtain stairs with approximately constant steps of a negligibly small order for truly straight lines, due to the limitations of our senses.

That is the solution for the infinitely small level. In practice, on an infinitely composed level, there is no basic cube that forms all others. There are no ideal smooth surfaces. On the infinitely composed level surfaces are exchangeable in transitional self-identical regions. The display of dimensions here is not static, but a dynamic one. There are six infinitely composed directions, forming three dimensions, in which the totality of all movements occurs. Hence, the dimensions by themselves are fluid, therefore space, which is composed of them, cannot be absolutely void. The other deal is relative void; as a matter of daily practice we obtain air for void, since its mechanical properties are too weak for us.

Geometry as a science could appear only because a number of objects possess a constancy of shapes, despite uninterrupted internal changes - electrons' rotation around nuclei in so-called solid bodies from the standpoint of quantum mechanics, gases of a peculiar genus; and in the absence of absolute void - liquids, whose flows, unlike those of a river, and akin to cytoplasm or blood, are directed on the establishment of a perfectly constant shape. Precisely on this level one can speak about curves and oblique lines, composing the shapes of animals, crystals etc.

In a two-dimensional totality, the maximal width of the moving value would equal 2 e infinitely small units. Otherwise, the units found opposite its middle cannot pass from the fore sub-totality to the rear one. On the other hand, the real value is infinitely composed, i.e. sufficiently greater than $2_{\mathrm{e}}$ (fig. 21).

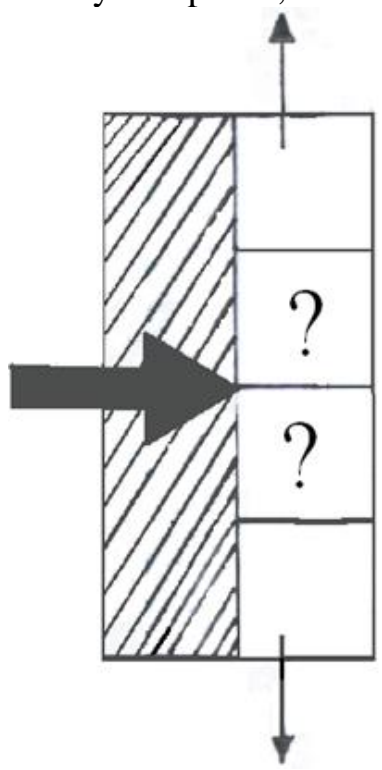

Figure 21. The problem of two-dimensional totality

Thus, on the infinitely small level, the third dimension provides the necessary condition for the movement of a value, wider than $2 \mathrm{e}$ (fig. 22). 
Nevertheless, the third dimension is infinitely composed as well, i.e. sufficiently greater than 2e. This means that no moving object may circumvent a given one. Nevertheless, the lesser values penetrate the given one without destructing it, which would be impossible in a two-dimensional totality. Thus, any moving object, including micro-particles, is represented by a penetrable net of its proper structures on an infinite totality of levels.

As far as concerns the fourth dimension, Minkowsky assumed that it is time. The scientist came to that conclusion from the statement of the theory of relativity, that both time and length are altered in an equal degree, but in opposite proportionality. Arguing the unity of space and time, Minkowsky equated the latter to ordinary dimensions of space (Minkowski, 1973, p. 169 - 171, 187), abstracting from the time its properties as currency and irreversibility. Furthermore, he proposed to represent the resting dot as a line, parallel to the axis of time; a uniformly moving point, as a section, inclined to this axis and its uneven motion as a curve (Ibid., p. 171). Thus, Minkowsky translates dynamics into statics. The author of the present article believes that this is connected to contradictions within the motion. As a result, it was rejected by the ancient Greek school of Eleatics, to which Parmenides and Zeno belonged.

On the other hand, as was shown above, all dimensions are dynamic. Hence, time is a component of all dimensions, and is not separate from them.

Therefore, the first dimension represents the direction of the movement of a given value; the second dimension, the being of the value; and the third, the way for the values of lesser significance. It is likely that the number of dimensions is manifested by these conditions. Nevertheless, it may be that the author missed some causes for additional dimensions. It could be noted also that the most flattened organisms have tentacles or a bending of the body in the third dimension. The same would be true if additional ones didpresent.

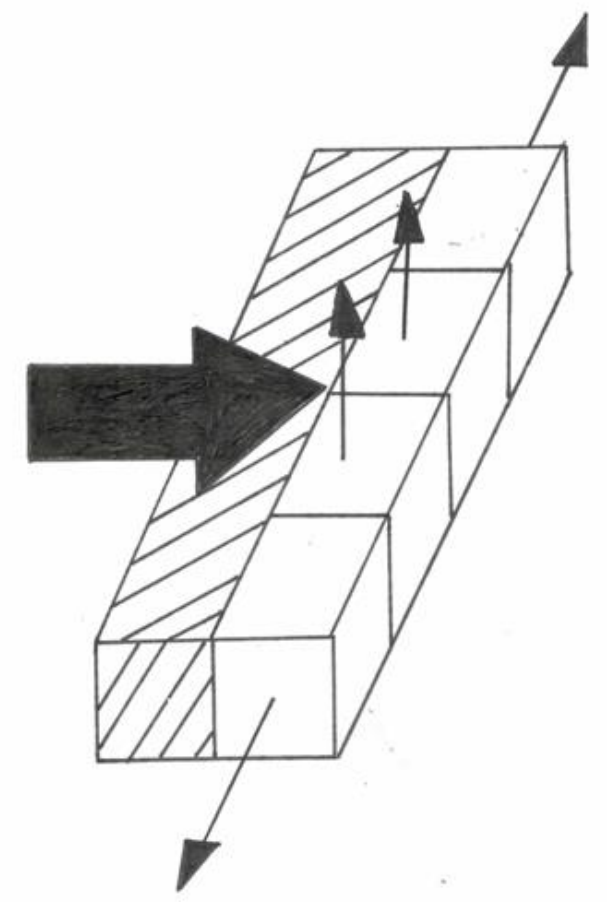

Figure 22. Conclusion of the third dimension 
As follows from both, the notion of curves and the metametric essentials, curved space, described by nonEuclidean geometries, cannot exist in reality. Non-Euclidean geometries should not be universal, but rather, specific cases of the Euclidean one, describing the behavior of basic lines drawn on a surface with particular figure.

\section{CONCLUSIONS}

Lobachevsky writes: "There are no straight or curved lines in nature, there are no planes and surfaces: we find in it only bodies, so that all the rest, formed by our imagination, exists only in theory."; "The notions do not yet contain the truth, which men want to prove, and which may be tested, like other physical laws, by experiments only, such as, for example, astronomic observations" (Lobachevsky, 1949, p. 147).

The first lemma, to which Nassir al-Din Tusi refers, differs from the above statement in introduction of segments, which are perpendicular to one of the considered lines, whereas relative to the other they form obtuse and acute angles. The geometer remarks that the line approaches the other from the side of the acute and removes from the side of obtuse angles (Kagan, 1955, p. 44). This condition corresponds to only one of the possible variants of the fifth postulate - when one of the inner angles is straight. In accordance with the fifth postulate, the angles may be chosen arbitrarily.

The statement that "Two straight lines cannot cross one another, if any third straight line crosses them at equal angles" Lobachevsky considers to be an axiom (number 7) (Lobachevsky, 1946, p. 81).

Inter alia, if one of the defined lines is perpendicular to the defining one, it does not incline nor decline from the line, declining towards it. This latter line passes an additional distance in the dimension, separating the defined lines. Hence, in the opposite order, a perpendicular line from a point to line, is shorter than an oblique line. In a more general case, a shorter segment, from a point on a straight line, has lesser inclination towards the other one, therefore, by a greater angle with the inner area of the defining line (fig. 23).
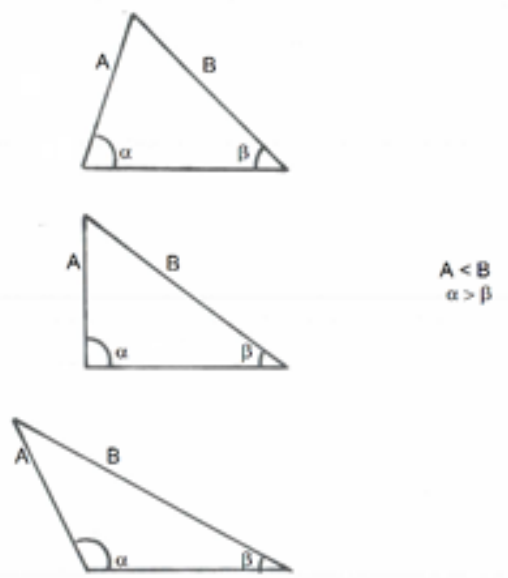

Figure 23. The relation between sides and angles

At the present time the term "negative angle" is applied to that angle, obtained by means of a clockwise rotation, and positive angle - that obtained by a counterclockwise rotation. I propose to call them "negative and positive turns" or, to be clearer, "counterclockwise and clockwise turns" and the term "negative angle" refers to the angle, obtained after passing the point of crossing. 
It is advisable to remember the words of the founder of the theory of relativity: "Euclidean geometry should be considered physical science, whose virtue should be shown by its application to sensuous perceptions."(Einstein, p. 206).

The Euclidean definition of parallel lines led to mysticism, while curves, declining from one another, are considered to be parallel only because they do not cross one another. An even more mystical sequence of the criterion of parallels is the notion of parallel worlds, which do not have common areas, but in all other aspects are the same. The voyage between them is miraculous, since it violates the established initial idea.

In metametry, parallel lines do not only not cross one another, they may be adjacent to one another. They are unifunctional dimensions forming others of a greater order, like the heights of interlines compose the height of a page.

\section{REFERENCES}

Aristotle. (1975). Metaphysics. Collected Works in 4 volumes, Vol. 1 (A.B. Kubitsky, Trans. from Ancient Greek). Moscow: Mysl press.

Beltrami, Eugenio. (1868). Teoria fondamentale degli spazii di curvatura constante. Annali. Di Mat., Series II. 2, 232 - 255). DOI:10.1007/BF02419615

Berkeley George. (1978). Writings, The Analyst: a Discourse addressed to an Infidel Mathematician. (Ye.S. Lagutin, Trans. from English). Moscow: Mysl press

Chattopadhyaya, D. (1981). What is Living and What is Dead in Indian Philosophy. (E.N. Anikeeva, et al., Transl. from English). Moscow: Progress

Chistiakov, V.D. (1973). Conversations about the geometry of Lobachevsky. Minsk: Vysheyshaya Shkola

Costoya, C. \& Viruel, A. (2014). Every finite group is the group of self-homotopy equivalences of an elliptic space. Acta Mathematica, 213(1), 49 - 62.

Courant, R. \& Robbins, H. (2001). What is Mathematics? An Elementary Approach to Ideas and Methods. (A.N. Kolmogorov, Transl. from English). Moscow: CCME

Dafermos, M., Holzegel, G. \& Rodnianski, I. (2019). The linear stability of the Schwarzschild solution to gravitational perturbations. Acta Mathematica, 222(1), 1-214

Democritus. (1970). Texts, translation, investigations. (S Ya. Lurie, Composition and translation). Leningrad: Nauka

Descartes. (1989). Writings in 2 volumes. Vol. . From Correspondence to M. Mersenn, Amsterdam, 15 of April 1630, 1619 - 1643. (Ya.A. Liatker, \& S.Ya. Sheynman-Topshtein, (Transl. from Latin and French) Moscow: Mysl press

Diadokhos, P. (2013). Commentary on the First Book of Euclid's "Essentials." (A.I. Schetnikov, Translation from Ancient Greek), Moscow: Dmitry Pozharsky University.

Einstein, A. (1967). Physics and reality. Articles, reviews, letters. Collection of Scientific Works in 4 volumes. Vol. 4. (Yu.A. Danilov, S.G. Suvorov, \& A.M. Frenk, Trans.). Moscow: Nauka press

Euclyd. (1948). Essentials. (D.D. Morduhay-Boltowsky, Trans. from Ancient Greek). Moscow-Leningrad: State Publishing House of Physical and Mathematical Literature 
Galilei, Galileo. (n.d.). Conversations and mathematical demonstrations, regarding two new branches of science that relate to mechanics and local movement of Signor Galileo Galilei Linceo, philosopher and first mathematician of his Serene Highness Great Duke of Toscana with application about centers of gravity of different bodiesWorks. Vol. I. (N.M. Televna, Transl. from Italian). Moscow - Leningrad: State Technic-Theoretic Publishing House, MCMXXXIV

Gassendi, P. (1966). Summa of the Philosophy of Epicurus. Writings in two volumes. Vol. 1 (A. A. Guterman, Trans. from Latin). Moscow: Mysl press

Kagan, V.F. (1955). Lobachevsky and his Geometry, Popular Essays. Moscow: State Editor for Technical and Theoretic Literature

Kant, Immanuel. (1964). Critique of Pure Reason. Writings in 6 volumes. Vol. 3. (N.O. Lossky, Trans. from German). Moscow: Mysl press

Cavalieri, B. (1977). Degrees of sums of indivisible lines. In Reader on the history of mathematics. (S.Ya. Lurie, Transl. from Latin, pp 49-50). Moscow: Prosveschenie

Kepler, I. (1977). The measurement of around with the help of indivisibles. In Reader on the history of mathematics. (G.N. Sveshnikov Trans. from Latin, pp 44-45). Moscow: Prosveschenie

Lobachevsky, N.I. (1946). Geometrical researches on the theory of parallel lines. Complete Work Collection. Vol. 1. In N.I. Lobachevsky. About essentials of geometry. (V.F. Kagan, Trans. from German). Moscow-Leningrad: State Publishing House of Physical and Mathematical Literature

Lobachevsky, N.I. (1949). New essentials of geometry with complete theory of parallel lines. Complete Work Collection. Vol. 2. Moscow-Leningrad: State Publishing House of Physical and Mathematical Literature

Engels, F. (1961). Anti-Dühring. In K. Marx, \& F Engels. Writings in 30-th volumes. Vol. 20. (Yu.I. Aihenvald, Trans. from German).

Maxwell, J.K. (1954). Collected Works on the Theory of the Electromagnetic Field: About Physical Power Lines. (Z.A. Tseitlin, Trans). Moscow: State Technic-Theoretic Publishing House

Minkowski, H. (1973). Space and Time. In The Principle of Relativity, Collected Works on the Special Theory of Relativity (Trans. from German). Moscow: Atomizdat

Nazarov F., Treil, S., \& Volberg, A. (2003). The Tb-theorem on non-homogeneous spaces. Acta Mathematica, 190(2), 151-239.

Newton, I. (n.d.) Four Letters from Sir Isaac Newton to Doctor Bentley, containing some Arguments in Proof of a Deity. London: printed for R. and J. Dodsley, Pall Mall MDCCLV.

Newton, I. (1952). Optics or a Treatise of the Reflections, Refractions, Inflections \& Colours of Light. NY: Dover Publications Inc. (Based on the fourth edition London, 1730).

Physical Encyclopedic Dictionary (1983). Moscow: Sovetskaya Encyklopediya.

Hawking, Stephen W. (1988). A Brief History of Time from the Big Bang to Black Holes. Toronto, NY, London, Sydney, Auckland: Bantam Books

Thomson, W. (Lord Kelvin). (1895). The Structure of Matter, Speeches and Popular Lectures. Resilience, Considered as a Peculiar Kind of Movement. (B.P. Weinberg, Trans. from English). Saint Petersburg: L. F. Panteleev's publishing house 